\title{
Classification of Epileptic Seizure EEG Based on Fully Connected Neural Network
}

\author{
Seok-Woo Jang ${ }^{1}$, Sang-Hong Lee ${ }^{2}$ \\ ${ }^{1}$ Dept. of Software, Anyang University, Republic of Korea, swjang7285@gmail.com \\ ${ }^{2}$ Dept. of Computer Science \& Engineering, Anyang University, Republic of Korea, shleedosa@ gmail.com
}

\begin{abstract}
This paper proposes a method to classify epileptic seizure EEG and normal EEG using Fully Connected Neural Network (FCNN). Wavelet transform (WT) was used in the first step to extract features to be used in FCNN. In the second step, 24 features were extracted from the wavelet coefficients generated in the first step using a frequency distribution and a frequency variation. FCNN showed a classification performance of $99.99 \%$ when classifying epileptic seizure EEG and normal EEG using these 24 features.
\end{abstract}

Key words : EEG signal, Epileptic seizure, FCNN, Wavelet transform.

\section{INTRODUCTION}

Electroencephalogram (EEG) records the electrical activity of the cerebral cortex and is used to measure or diagnose brain-related sleep conditions, brain-computer interface (BCI), and disease [1-4]. In particular, in the case of epilepsy patients, monitoring of EEG is an important factor for confirming the status of epilepsy patients. Epilepsy refers to a chronic disease caused by repeated seizure symptoms causing convulsions and consciousness disorder, despite no physical abnormalities. Artificial intelligence techniques using brain waves have been studied to detect and diagnose such epilepsy [5-9].

In order to extract the features of the EEG signal, a wavelet transform (WT), which is a Fourier transform and a time-frequency analysis technique, is preferred, and is used for classification of epileptic seizure by grafting to a fuzzy neural network [5-7].

Polat [8] classified the epileptic seizure EEG using Fourier transform. Polat extracted 129 factor values through spectral analysis of Fourier transform. The extracted 129 factor values were reduced to 5 using principal component analysis (PCA), and these values were used as features. However, the drawback of this study is that it can be used for classification experiments of epileptic seizure because it needs to extract many factor values of 129 and uses principal component analysis to reduce the number of features, but it is applicable to the classification of real-time epileptic seizure EEG using EEG measuring devices. The disadvantage is that this is impossible. Subasi [6] extracted features based on coefficients generated through wavelet transform. A classification model called mixture of expert (ME) was used for the extracted feature.

In this paper, normal EEG and epileptic seizure EEG were classified from EEG data used by Andrzejak et al [10] using the Fully Connected Neural Network (FCNN), which shows excellent performance in classification performance. In the first step, wavelet transform was used to extract features to be used in FCNN. In the second step, 24 features were extracted from the wavelet coefficients generated in the first step using the frequency distribution and the amount of frequency variation. FCNN showed a classification performance of 99.99\% when classifying normal EEG and epileptic seizure EEG using these 24 features.

\section{DATA AND PREPROCESSING}

In this paper, wavelet transforms (WT) are performed in the pre-processing process using EEG data as an input in Figure 1. In the feature extraction process after the pre-processing, the feature is extracted using the frequency distribution and frequency variation for the wavelet transformed EEG signal, and the normal EEG and epileptic seizure EEG are classified using the FCNN.

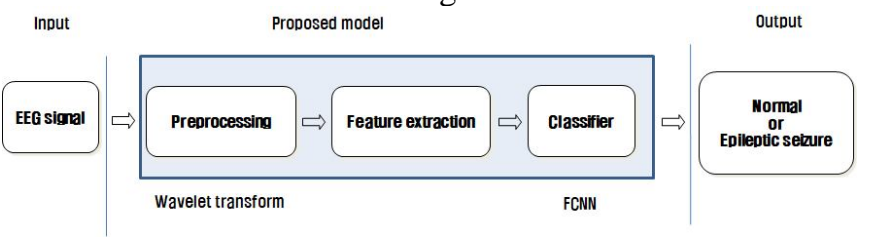

Figure 1: Proposed model

\subsection{Data}

In this paper, normal EEG and epileptic seizure EEG were classified using EEG data used by Andrzejak et al [8]. The experimental data used by Andrzejak et al is divided into 5 experimental groups (A, B, C, D, and E). Each experimental group contains 100 single-channel EEG segments. In this paper, experiment data were accomplished using the experimental groups $\mathrm{A}$ and $\mathrm{E}$ used by Subasi [6]. 
Experimental group A is a normal EEG collected through healthy subjects and experimental group $\mathrm{E}$ is an epileptic seizure EEG collected through subjects with epilepsy symptoms. Table 1 shows the experimental groups used in this paper. Each signal constituting the experimental group consists of 512 signals.

Table 1: Numbers of training and test sets

\begin{tabular}{|l|c|c|c|}
\hline \multicolumn{1}{|c|}{ Class } & Train set & Test set & Total set \\
\hline $\begin{array}{l}\text { Epileptic } \\
\text { seizure }\end{array}$ & 500 & 300 & 800 \\
\hline Normal & 500 & 300 & 800 \\
\hline Total & 1000 & 600 & 1600 \\
\hline
\end{tabular}

\subsection{Wavelet Transforms}

In this paper, the wavelet transform was performed as shown in Figure 2. Using the experimental data used by Andrzejak et al, a single wavelet consisting of 512 signals was transformed into Haar wavelet with a scale level of 5 as shown in Figure 2.

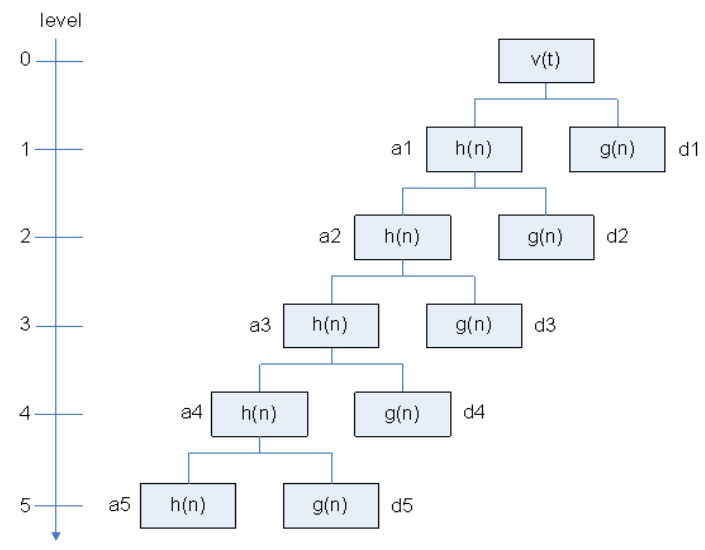

Figure 2: Wavelet transform with scale level 5

Figure 3 and 4 show the values obtained by performing the Haar wavelet transform. Figure 3 shows an example of a Haar wavelet transformed normal EEG and Figure 4 shows an example of a Haar wavelet transformed epileptic seizure EEG. The frequency of the experimental data used by Andrzejak et al is $173.6 \mathrm{~Hz}$.

\subsection{Feature Extraction}

The features to be used in this paper were extracted as follows. From (1) to (4), the feature extraction method used in Subasi [6] was described. In addition, in this paper, the features described in (5) were newly added and tested.

(1) The average value of absolute values for all coefficients in each level

(2) The average value obtained by squaring all coefficients in each level

(3) Standard deviation of all coefficients in each level
(4) The ratio of the absolute value of the average value to all coefficients within the level between adjacent levels.

(5) Median of all coefficients in each level

The features (1) and (2) mentioned above refer to the frequency distribution for the EEG signal. Also, the features (3) and (4) indicate the frequency variation [6]. In this paper, 24 features were extracted using frequency domains a5 and $\mathrm{d} 2 \sim \mathrm{d} 5$. In the frequency domain of $\mathrm{d} 2$ to $\mathrm{d} 5$, all the features from (1) to (5) mentioned above were used, and in the frequency domain of a5, the remaining four except for (4) were used because there was no adjacent level.

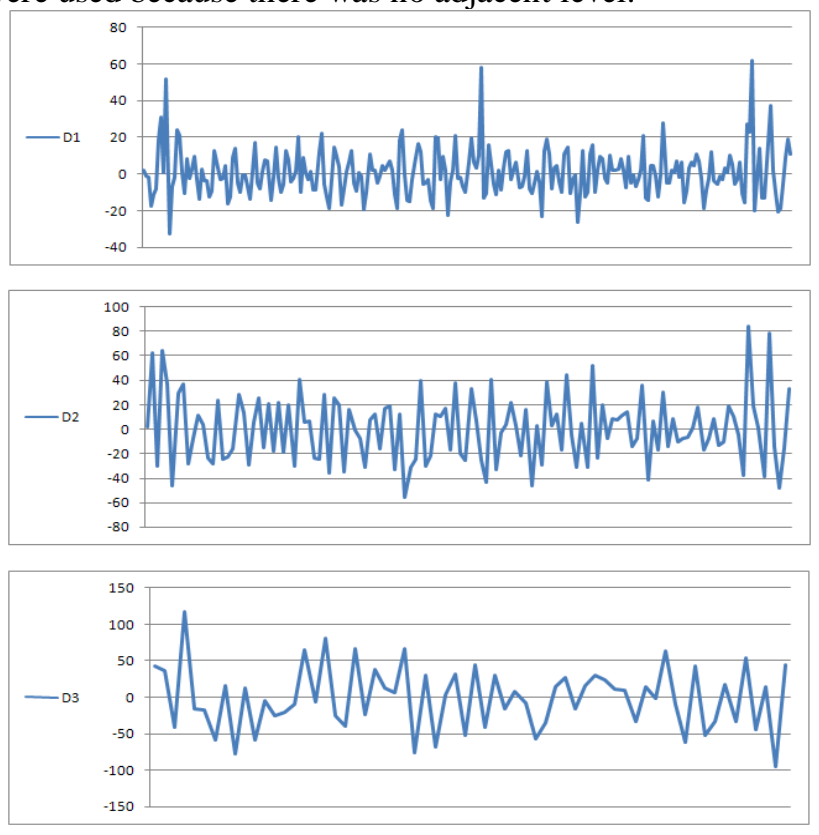

Figure 3: Examples of wavelet coefficients of normal EEG

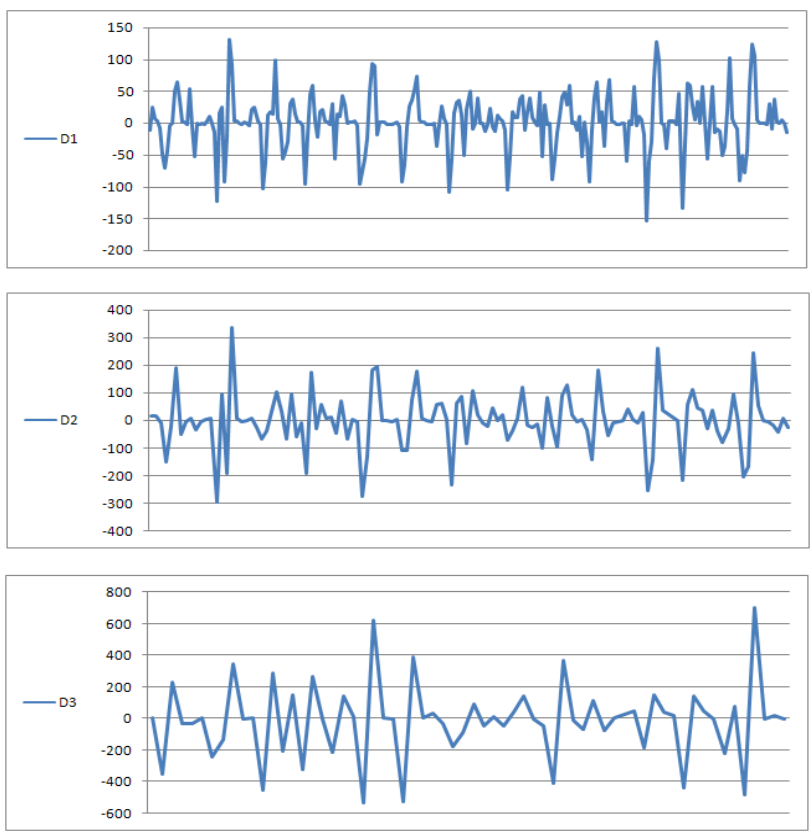

Figure 4: Examples of Wavelet coefficients of epileptic seizure EEG 


\section{Fully CONNECTED NeURAL NETWORK (FCNN)}

A fully connected neural network (FCNN) is a sort of artificial neural network that has an architecture so that all nodes or neurons in one layer connect to neurons in the next layer. This type of algorithm is usually applied to some type of data, but in reality this type of network has some problems in terms of image recognition and classification. Such networks are computationally intense and prone to overfitting. Figure 5 shows multi-layers of FCNN used in this experiment.

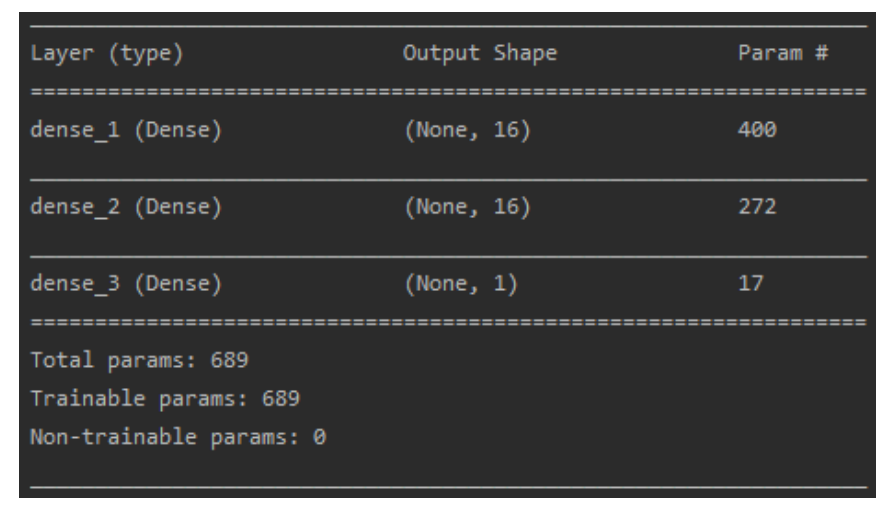

Figure 5: Multi-layers of FCNN

\section{EXPERIMENTAL RESULTS}

In this paper, normal EEG and epileptic seizure EEG were classified using EEG experimental data used by Subasi [6]. FCNN generates coefficients from wavelet transforms a5 and d2 to d5 in Fig. 3 and 4 and learns them using 24 features extracted using frequency distribution and frequency variation.

$$
\begin{gathered}
\text { Sensitivity }=\frac{T P}{T P+F N} \times 100 \\
\text { Specificity }=\frac{T N}{T N+F P} \times 100 \\
\text { Accuracy }=\frac{T P+T N}{T P+F N+T N+F P} \times 100
\end{gathered}
$$

Table 2: Confusion matrix of performance results

\begin{tabular}{|c|c|c|}
\hline Class & \multicolumn{2}{|c|}{ Results } \\
\hline \multirow{2}{*}{$\begin{array}{c}\text { Epileptic seizure } \\
(300)\end{array}$} & TP & FN \\
\cline { 2 - 3 } & 491 & 9 \\
\hline $\begin{array}{c}\text { Normal } \\
(300)\end{array}$ & FP & TN \\
\cline { 2 - 3 } & 0 & 500 \\
\hline
\end{tabular}

Table 3: Comparisons of performance results

\begin{tabular}{|c|c|c|c|}
\hline & Sensitivity & Specificity & Accuracy \\
\hline Subasi [6] & $95 \%$ & $94 \%$ & $94.5 \%$ \\
\hline FCNN & $98.2 \%$ & $100 \%$ & $99.1 \%$ \\
\hline
\end{tabular}

Table 2 and Table 3 show the classification performance. TP (True Positive) means that a signal that is not a normal EEG is classified as a signal that is not a normal EEG, and TN (True Negative) means that a normal EEG is classified as a normal EEG. In addition, FP (False Positive) means that the epileptic seizure EEG is classified as a signal that is not an epileptic seizure EEG, and FN (False Negative) means that a signal that is not an epileptic seizure EEG is classified as an epileptic seizure EEG. The sensitivity, specificity, and accuracy obtained in Table 3 are defined as in Equation (1). As shown in Table 3, the results of $100 \%, 96 \%$, and $98 \%$ are all improved in sensitivity, specificity, and accuracy than the results of Subasi [6].

\section{CONCLUSION}

In this paper, 24 features were extracted from the coefficients generated using wavelet transform using statistical techniques of frequency distribution and frequency variation. In addition, we present a method for automatic detection of normal EEG and epileptic seizure EEG from FCNN-based EEG signals using these 24 features. Through the method proposed in this paper, it is possible to implement a system capable of classifying normal EEG and epileptic seizure EEG by measuring brain waves in real time. It shows the classification results of $98.2 \%, 100 \%$, and $99.1 \%$ with improved sensitivity, specificity, and accuracy than the results of Subasi [6].

\section{ACKNOWLEDGEMENT}

This work was supported by the National Research Foundation of Korea (NRF) grant funded by the Korea government (MSIT) (No. NRF-2019R1F1A1055423).

Corresponding Author: Sang-Hong Lee

(shleedosa@gmail.com)

\section{REFERENCES}

1. Fan Zhang, Hang Yu, Jie Jiang, Zhangye Wang, Xujia Qin, Brain-computer control interface design for virtual household appliances based on steady-state visually evoked potential recognition, Visual Informatics, Vol.4, pp.1-7, 2020.

https://doi.org/10.1016/j.visinf.2019.12.001

2. M. Kemal Kiymik, Mehmet Akin, Abdulhamit Subasi, Automatic recognition of alertness level by using wavelet transform and artificial neural network, Journal of Neuroscience Methods, Vol.139, pp.231-240, 2004.

https://doi.org/10.1016/j.jneumeth.2004.04.027

3. Kaushalya Kumarasinghe, Nikola Kasabov, Denise Taylor, Deep learning and deep knowledge representation in Spiking Neural Networks for 
Brain-Computer Interfaces, Neural Networks, Vol.121, pp. 169-185, 2020.

https://doi.org/10.1016/j.neunet.2019.08.029

4. Abdulmajeed Alsufyani, Analyses of The P300 Event

Related Potentials in EEG Signals Using Shape-based Kernel: Fixed and Random Analyses Approach, International Journal of Emerging Trends in Engineering Research, Vol.8, pp.476-490, 2020.

https://doi.org/10.30534/ijeter/2020/36822020

5. Amal Fouad, Hossam M. Moftah, Hesham A. Hefny, MRI Brain Cancer Diagnosis Approach Using Gabor Filter and Support Vector Machine, International Journal of Emerging Trends in Engineering Research, Vol.7, pp.907-914, 2019.

https://doi.org/10.30534/ijeter/2019/297122019

6. Abdulhamit Subasi, EEG signal classification using wavelet feature extraction and a mixture of expert model, Expert Systems with Applications, Vol.32, pp.1084-1093, 2007.

https://doi.org/10.1016/j.eswa.2006.02.005

7. Adeli, H., Zhou, Z., \& Dadmehr, N., Analysis of EEG records in an epileptic patient using wavelet transform, Journal of Neuroscience Methods, Vol.123, pp.69-87, 2003.

8. Kemal Polat and Salih Güneş, Artificial immune recognition system with fuzzy resource allocation mechanism classifier, principal component analysis and FFT method based new hybrid automated identification system for classification of EEG signals, Expert Systems with Applications, Vol.34, pp.2039-2048, 2008.

https://doi.org/10.1016/j.eswa.2007.02.009

9. Srinivasan V, Eswaran C, Sriraam N, H., Approximate Entropy based Epileptic EEG detection using Artificial Neural Networks, IEEE Transactions on Information Technology in Biomedicine, Vol.11, pp.288-295, 2007.

10. Andrzejak, R. G., Lehnertz, K., Mormann, F., Rieke, C., David, P., and Elger, C. E., Indications of nonlinear deterministic and finite dimensional structures in time series of brain electrical activity: Dependence on recording region and brain state, Physical Review E, 64, 061907, 2001.

https://doi.org/10.1103/PhysRevE.64.061907 